\title{
A sirtuin in the African trypanosome is involved in both DNA repair and telomeric gene silencing but is not required for antigenic variation
}

OnlineOpen: This article is available free online at www.blackwell-synergy.com

Sam Alsford, Taemi Kawahara, Cyril Isamah and David Horn*

London School of Hygiene and Tropical Medicine, Keppel Street, London, WC1E 7HT, UK.

\section{Summary}

Silent information regulator 2 (Sir2)-related proteins or sirtuins function as $\mathrm{NAD}^{+}$-dependent deacetylases or ADP ribosylases that target a range of substrates, thereby influencing chromatin structure and a diverse range of other biological functions. Genes encoding three Sir2-related proteins (SIR2rp1-3) have been identified in the parasitic trypanosomatids, early branching protozoa with no previously reported transcriptional silencing machinery. Here we show that, in the mammalian-infective bloodstream-stage of the African trypanosome, Trypanosoma brucei, SIR2rp1 localizes to the nucleus while SIR2rp2 and SIR2rp3 are both mitochondrial proteins. The nuclear protein, SIR2rp1, controls DNA repair and repression of RNA polymerase I-mediated expression immediately adjacent to telomeres. Antigenic variation, however, which involves the silencing and Pol I-mediated transcriptional switching of subtelomeric variant surface glycoprotein genes, continues to operate independent of SIR2rp1.

\section{Introduction}

SIR2-related NAD+-dependent (class III) deacetylases or sirtuins (reviewed in Blander and Guarente, 2004) are found in organisms from all biological kingdoms (Frye, 2000). Saccharomyces cerevisiae Sir2, the founding member of the group, is a histone deacetylase (reviewed in Rusche et al., 2003) involved in a range of chromatinmediated processes; namely, gene silencing at telomeres and mating-type loci, DNA repair (Tsukamoto et al., 1997;

\footnotetext{
Accepted 30 November, 2006. *For correspondence. E-mail david. horn@ @ Ishtm.ac.uk; Tel. (+44) 0207927 2352; $\quad$ Fax (+44) 020 76368739 .

Re-use of this article is permitted in accordance with the Creative Commons Deed, Attribution 2.5, which does not permit commercial exploitation.
}

Martin et al., 1999), suppression of recombination within ribosomal DNA (rDNA; Gottlieb and Esposito, 1989), DNA replication (Pappas et al., 2004), chromosome stability (Holmes et al., 1997) and plasmid segregation (Longtine et al., 1993). It is now recognized, however, that sirtuins remove acetyl groups from lysines in nuclear, cytosolic and mitochondrial substrates (reviewed in Blander and Guarente, 2004). For example, Sir2 orthologues regulate the function of the p53 transcription factor (Luo et al., 2001; Vaziri et al., 2001) and acetyl CoA synthetase (Starai et al., 2002); and TAF,68 (Muth et al., 2001), tubulin (North et al., 2003) and the archaeal protein, Alba (Bell et al., 2002) are among other known substrates. Sir2 orthologues can also modify small molecules, as in the case of cobB which functions in the cobalamine biosynthetic pathway (Tsang and Escalante-Semerena, 1998), and they have now also been linked to ageing in a range of systems (reviewed in Blander and Guarente, 2004). Thus, sirtuins modulate gene silencing, DNA repair and energy metabolism through post-translational modification of a variety of factors. During the Sir2 deacetylation reaction, acetyl-lysine and $\mathrm{NAD}^{+}$are converted to lysine, nicotinamide and O-acetyl-ADP-ribose (Tanner et al., 2000). Thus, the full range of functions assigned to this protein family may be explained by small-molecule substrates and products as well as the array of protein substrates.

Trypanosoma brucei, a protozoan that branched early from the eukaryal lineage, is the causative agent of African sleeping sickness. The parasite undergoes antigenic variation in the mammalian host, a process that requires the maintenance of several hundred subtelomeric variant surface glycoprotein (VSG) genes in a transcriptionally silent state (reviewed in Horn and Barry, 2005). Histone acetylation operates in T. brucei (Janzen et al., 2006) and one Sir2 orthologue, SIR2rp1, has been characterized in insect stage cells indicating a role in DNA repair through $\mathrm{NAD}^{+}$-dependent $\mathrm{ADP}$-ribosylase and deacetylase activities (Garcia-Salcedo et al., 2003). Here, we report characterization of the T. brucei sirtuins in bloodstream form cells. In these cells, SIR2rp1 localizes to the nucleus while the other two proteins localize to the parasite's single mitochondrion. Although the nuclear 
protein is involved in telomeric silencing it is not required for VSG gene silencing or antigenic variation.

\section{Results}

Three sirtuins expressed in T. brucei

Three trypanosomatid genomes have been sequenced (see Ivens et al., 2005). Among the sequences, genes encoding three proteins were identified in T. brucei, and Leishmania major and two in Trypanosoma cruzi related to S. cerevisiae Sir2. For comparison, the human genome encodes seven Sir2 orthologues and S. cerevisiae encodes Sir2 plus four paralogues. The trypanosomatid genes were designated SIR2-related proteins SIR2rp1-3 and it is SIR2rp2 that is not represented in the T. cruzi genome (see table S4 in Ivens et al., 2005). Phylogenetic analysis places SIR2rp1 in a group with ScSir2, HsSIRT1 and HsSIRT2, while SIR2rp2 and SIR2rp3 are more closely related to bacterial proteins and HsSIRT4 and HsSIRT5 respectively (Fig. 1A). Although all trypanosomatid SIR2rp's lack the N-terminal extension of ScSir2 that is required for nucleolar localization (Cockell et al., 2000), they all contain a full catalytic domain (T. brucei proteins illustrated in Fig. 1B). The SIR2rp1 proteins have Ser-rich motifs towards the C-terminus and both SIR2rp2 proteins contain two insertions, the larger of which aligns with an insertion in the HsSIRT4 and the Streptomyces coelicolor proteins (see Fig. 1). All eight trypanosomatid proteins have a $\mathrm{Zn}^{2+}$ binding motif of the $\mathrm{CX}_{2} \mathrm{CX}_{20} \mathrm{CX}_{2} \mathrm{C}$ type but one of the Cys residues is absent in SIR2rp3 in all three trypanosomatids (see T. brucei proteins aligned in Fig. 1C), a feature shared with the HsSIRT5 and the Salmonella enterica proteins (see Fig. 1A). These findings indicate that each of the three SIR2rp's has similar features regardless of the trypanosomatid under consideration. We have focused on the T. brucei SIR2rp's found on chromosomes VII, VIII and IV encoding proteins with predicted molecular mass of approximately 38.5, 33.6 and $27 \mathrm{kDa}$ respectively.

We sequenced all three T. brucei genes from the Lister 427 strain and compared the sequences to the T. brucei genome reference strain (TREU 927/4). Each predicted protein differed at only two positions as follows; SIR2rp1, I161M and S281N; SIR2rp2, Q61R and D304G; SIR2rp3, R6G and D132E. All mature T. brucei mRNAs possess an identical 5'-spliced leader (SL) sequence added at a specific AG-dinucleotide, splice acceptor (SA). Using reverse transcription polymerase chain reaction (RT-PCR), we confirmed that all three genes are expressed in bloodstream form cells (data not shown) and identified trans-splicing sites, 198, $115 / 67$ and $23 \mathrm{nt}$ upstream of the start codon for
SIR2rp1-3 respectively. We also detected SIR2rp1 mRNA by Northern blot analysis using polyA ${ }^{+}$mRNA, revealing a transcript of approximately $1.8 \mathrm{~kb}$ in both mammalian bloodstream and insect mid-gut life cycle stages (data not shown).

\section{SIR2RP subcellular localization}

To gain insight into the T. brucei cellular compartment where each protein acts, we expressed inducible, tagged (cMyc or eGFP) versions of each protein in bloodstream form cells. In the absence of Tet, N-terminal cMyc-tagged mycSIR2rp1 was expressed at a low-level while Tet increased expression significantly (Fig. 2A).

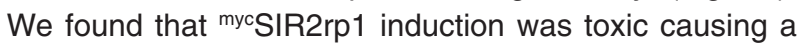
significant decrease in cell growth within $48 \mathrm{~h}$ (Fig. 2B). SIR2rp1 was previously reported to be a nuclear protein in insect-stage cells (Garcia-Salcedo et al., 2003). Analysis of fixed cells indicated a weak, but specific signal throughout the nucleus when ${ }^{m y}$ SIR2rp1 was expressed at a low level, while increased expression caused the protein to accumulate in the cytoplasm (Fig. 2C). Other cultures did not display growth defects following Tet addition (data not shown) indicating that it is increased mycSIR2rp1 expression and/or accumulation in the cytoplasm that is toxic.

Phylogenetic analysis indicated that trypanosomatid SIR2rp2 and SIR2rp3 are related to bacterial proteins and, respectively, the human mitochondrial proteins, HsSIRT4 and HsSIRT5 (Fig. 1A and see Michishita et al., 2005). Putative N-terminal signal sequences are indicated in LmSIR2rp2 and TbSIR2rp3 in the genome sequence database (http://www.genedb.org/). We therefore cloned SIR2rp2 and SIR2rp3 genes in a C-terminal GFP-tagging vector. Analysis of fixed cells expressing either SIR2rp2 ${ }^{\text {GFP }}$ or SIR2rp3 ${ }^{\text {GFP }}$ revealed GFP signals reminiscent of the single mitochondrion found in trypanosomes. We costained these cells with Mitotracker Red and merged images revealed almost total colocalization, indicating that both SIR2rp2 and SIR2rp3 are indeed mitochondrial proteins (Fig. 3B). When we expressed either of these proteins with an N-terminal tag we observed cytoplasmic localization of the full-length protein (data not shown) consistent with the idea that these tags interfere with $\mathrm{N}$-terminal mitochondrial import signals.

\section{SIR2rp1 influences DNA repair in bloodstream form cells}

SIR2rp1 is the only nuclear sirtuin in T. brucei (Fig. 2, and also see Fig. 3). To explore the role of this protein, we targeted the cognate gene for disruption in bloodstream form T. brucei cells. The SIR2rp1 gene is a 'single copy' 
A

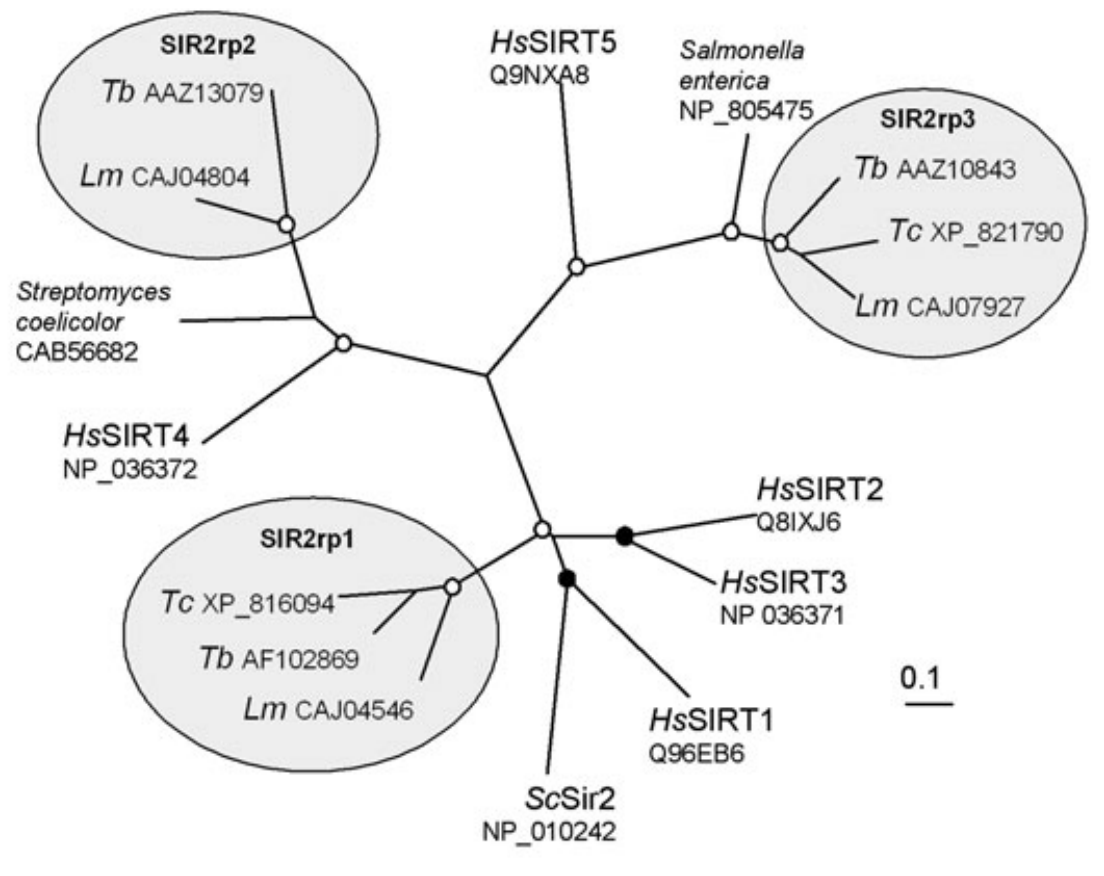

ScSIR2

TbSIR2rp1

TbSIR2rp2

TbSIR2rp3

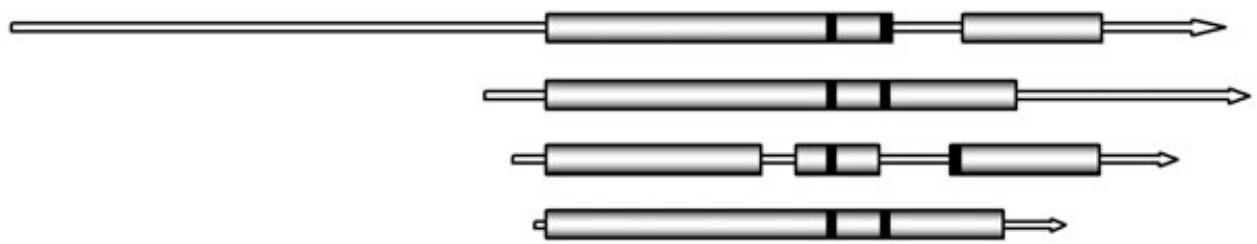

100 amino acids

Core deacetylase domains

C

SCSIR2 256 RKILVLTGAGVSTSL TbSIR 2 rp 130 TKIFVMVGAGISVAAGI PDFRSPHTGLYAKLSRYNLNSEEDAESLPLLRQQPSVFYNILM 59 TbSIR2rp2 15 QRCVILTGAGCSTESGVPDYRG-PNGLYRRPNFVPLTR---QVFLSGSEHOKRYWARSMF 70 TbSIR2rP3 6 BMIAILTGAGISAESGISTFRD-QNELWENHRVEDVCTFAAFLKQPTVVQR---FYNERR 61

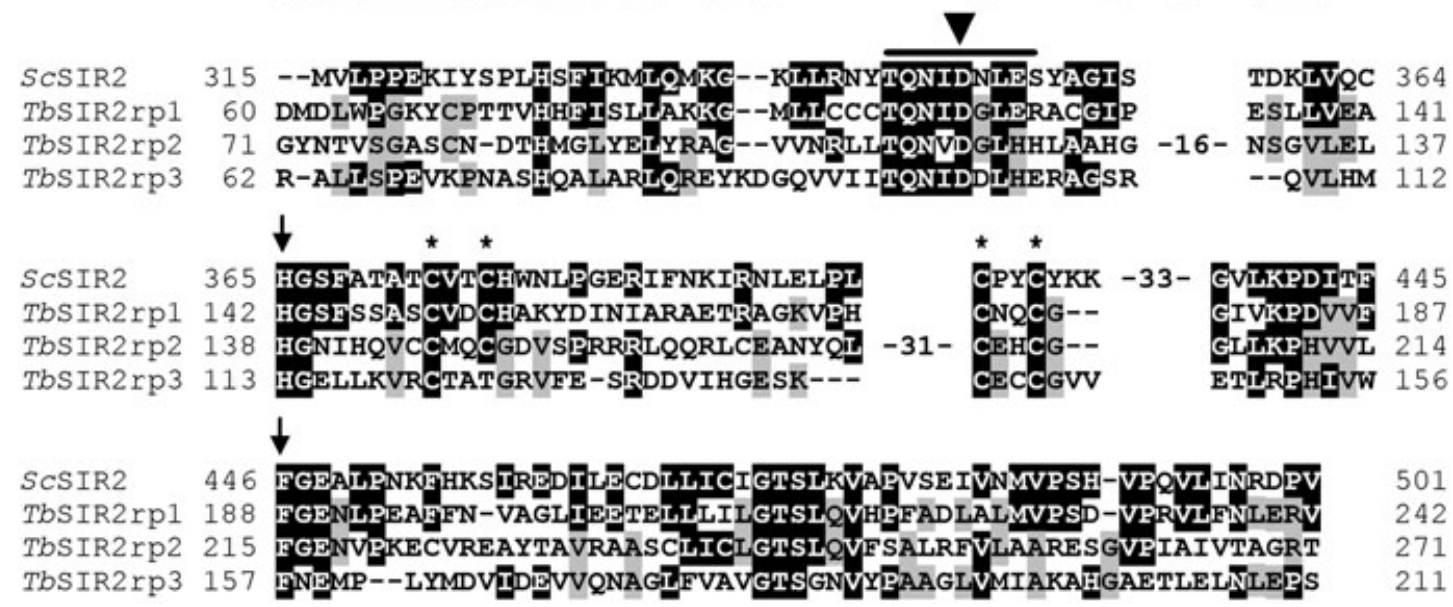


Fig. 1. Sirtuins in trypanosomatids.

A. The trypanosomatid SIR2rp's were compared to other Sir2 orthologues identified by BLAST analysis. The unrooted neighbour-joining tree was generated using Clustal $1.8 \times$ and TreeView. Branching confidence based on bootstrap replicas is indicated where excellent $(>99 \%$, open circle) or very good (>90\%, closed circles). Trypanosomatid SIR2rp's are indicated in shaded ovals. Tb, T. brucei; Tc, T. cruzi; Lm, L. major, Hs, Homo Sapien; Sc, S. cerevisiae.

B. Schematic representation of the predicted T. brucei proteins compared with S. cerevisiae Sir2. The location of Cys residues that form the $\mathrm{Zn}^{2+}$-binding motif are indicated (black bars).

C. The core deacetylase domains from the T. brucei SIR2rp's were aligned with the equivalent region from ScSir2. Residues that are identical between ScSir2 and any of the T. brucei proteins are white on a black background. Other residues shared among the $T$. brucei proteins are on a grey background. Asterisks indicate a putative $\mathrm{Zn}^{2+}$-binding motif. Bars above the sequence indicate the location of conserved motifs used to design PCR primers (see Experimental procedures). Arrows and arrowheads indicate critical catalytic or NAD+-binding residues respectively (see Imai et al., 2000; Min et al., 2001; Chang et al., 2002).

gene and T. brucei is diploid so we assembled a pair of constructs with different selectable markers. The constructs were used to disrupt residues 1-285 (see Fig. 4A). PCR (data not shown) and Southern analysis (Fig. 4A) indicated that the gene was disrupted as intended. Features that were indistinguishable between wild type and sir2rp1 null mutants (data not shown) include growth rate in culture, differentiation to the insect-stage with concomitant inactivation of VSG expression and tubulin acetylation status, as assessed using C3B9 antibodies (Sasse and Gull, 1988) on Western blots. We also disrupted the SIR2rp2 and SIR2rp3 genes. These mutant strains displayed no growth or differentiation defects (data not shown).
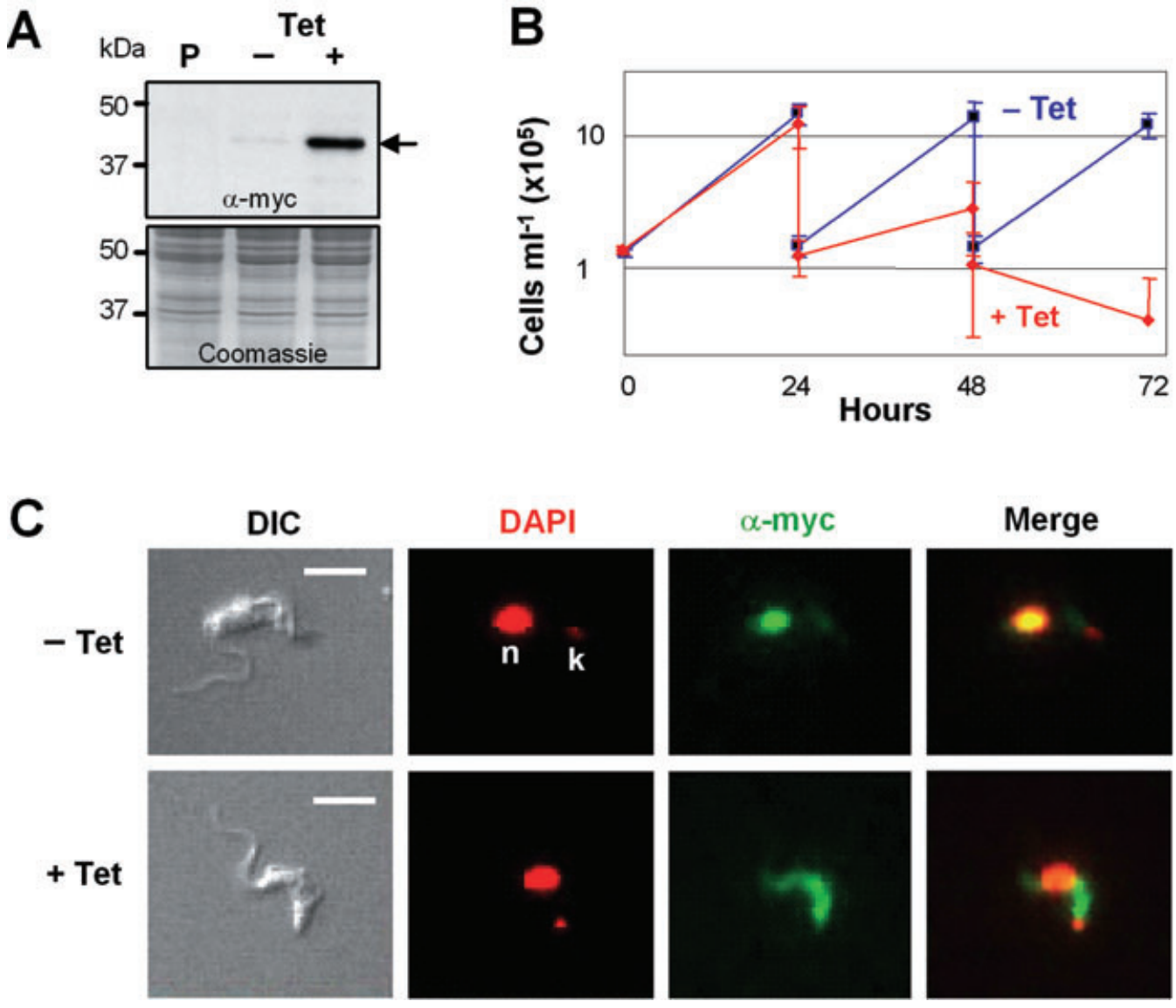

Fig. 2. SIR2rp1 localizes to the nucleus.

A. Western blotting with anti-cMyc and cell-lysates from parental $(P)$, and a representative ${ }^{m y c}$ SIR2rp1 cell line before $(-)$ and, after induction (+ ; $1 \mu \mathrm{g} \mathrm{ml}^{-1}$ tetracycline for $24 \mathrm{~h}$ ). The arrow indicates ${ }^{\text {myc }}$ SIR2rp1 which is expressed at a low level prior to induction. Bottom panel: Coomassie-stained gel as a loading control.

B. Growth curves generated from four independent ${ }^{\text {myc} S I R 2 r p 1 ~ c e l l ~ l i n e s ~ i n ~ t h e ~ p r e s e n c e ~ o r ~ a b s e n c e ~ o f ~ T e t . ~ C u l t u r e s ~ w e r e ~ d i l u t e d ~ t o ~} 10^{5}$ cells per $\mathrm{ml}$ as appropriate every $24 \mathrm{~h}$. Error bars: one standard deviation.

C. Immunofluorescence detection of mycSIR2rp1 (green). ${ }^{m y c}$ SIR2rp1 expressed at a low level localizes to the nucleus (upper panels, detected by long exposure) or the cytoplasm when expression level increases (lower panels). DNA counterstained with DAPI (false coloured in red); $k$, kinetoplast; $\mathrm{n}$, nucleus. Scale bar: $5 \mu \mathrm{m}$. 
A

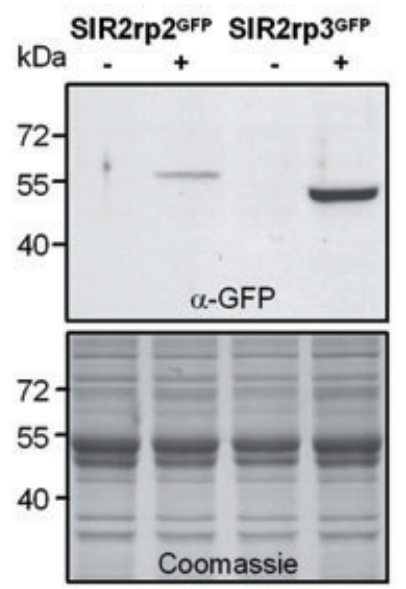

B

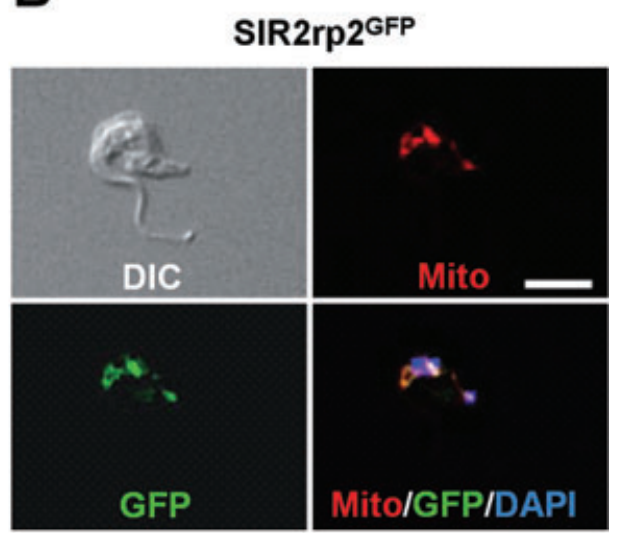

SIR2rp3 ${ }^{\text {GFP }}$

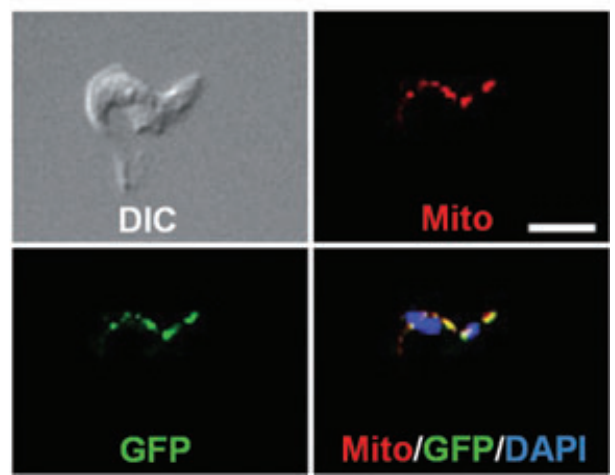

Fig. 3. SIR2rp2 and SIR2rp3 are mitochondrial proteins.

A. Western blotting with anti-GFP and cell-lysates from representative SIR2rp2 ${ }^{\text {GFP }}$ and SIR2rp3 ${ }^{\text {GFP }}$ cell lines before (-) and after induction (+ ; $1 \mu \mathrm{g} \mathrm{ml}^{-1}$ tetracycline for $24 \mathrm{~h}$ ). Bottom panel: Coomassie-stained gel as a loading control.

B. Immunofluorescence detection of GFP (green) in fixed cells revealed mitochondrial localization of SIR2rp2 ${ }^{\text {GFP }}$ and SIR2rp3 ${ }^{\text {GFP }}$ MitoTracker CMXRos (Mito) labelling (red). DNA counterstained with DAPI (blue). Scale bars: $5 \mu \mathrm{m}$.
Kluveromyces lactis sir2 mutants display hypersensitivity to DNA targeting drugs (Chen and Clark Walker, 1994) and assays with the DNA-damaging agent, methanesulphonic acid methyl ester (MMS) indicated that SIR2rp1 is involved in DNA repair in insect stage T. brucei (Garcia-Salcedo et al., 2003). We first confirmed a role for SIR2rp1 in DNA repair in insect stage sir2rp1 null mutants (data not shown) and then applied the MMS assay to bloodstream form cells where the $I_{50}$ is about 10-fold lower than for the insect stage (see Proudfoot and McCulloch, 2005). Bloodstream form sir2rp1 null mutants were more susceptible to MMS than wild-type cells (Fig. 4B). To confirm that increased MMSsensitivity was a consequence of SIR2rp1 deficiency, sir2rp1 cells were reconstituted with an expression vector which restored SIR2rp1 expression (Fig. 4B, inset). This was achieved by targeting a constitutively expressed ${ }^{m y c}$ SIR2RP1 gene to different rDNA spacer loci which support distinct expression levels (see Alsford et al., 2005). Only strains expressing SIR2rp1 at a subtoxic level (see Fig. 2) could be generated using this strategy. Restored SIR2rp1 expression reversed the increased MMS-hypersensitivity of sir2rp1 null mutants (Fig. 4B) indicating a role in DNA repair in both major life cycle stages of T. brucei.

\section{SIR2rp1 influences telomeric silencing but is not} required for antigenic variation

Telomeric gene silencing has been demonstrated in mammalian cells, in yeast, Plasmodium falciparum and T. brucei (see Glover and Horn, 2006), and silencing is known to rely upon Sir2 in yeast (reviewed in Rusche et al., 2003) and a sirtuin in P. falciparum (reviewed in Figueiredo and Scherf, 2005). In addition, T. brucei SIR2rp1 was reported previously to be associated with telomeric DNA (Garcia-Salcedo et al., 2003). We first tested SIR2rp1 in a telomeric silencing assay in S. cerevisiae. SIR2rp1 failed to complement a Scsir2 defect (strain UCC2, data not shown) but overexpression of SIR2rp1 and SIR2rp3 disrupted silencing (Fig. 5). This dominant negative effect, which is similar to that observed for the yeast cytosolic Sir2 paralogue, HST2 (Perrod et al., 2001), could reflect competition with Sir2 for a substrate or ligand, such as $\mathrm{NAD}^{+}$, or production of nicotinamide, a Sir2 inhibitor (Bitterman et al., 2002). One possible explanation for a lack of activity for SIR2rp2 may be a requirement for mitochondrial import and the associated processing as is the case with human SIRT3 (Schwer et al., 2002). Although the yeast analysis demonstrated a clear activity for SIR2rp1, it did not provide 
A
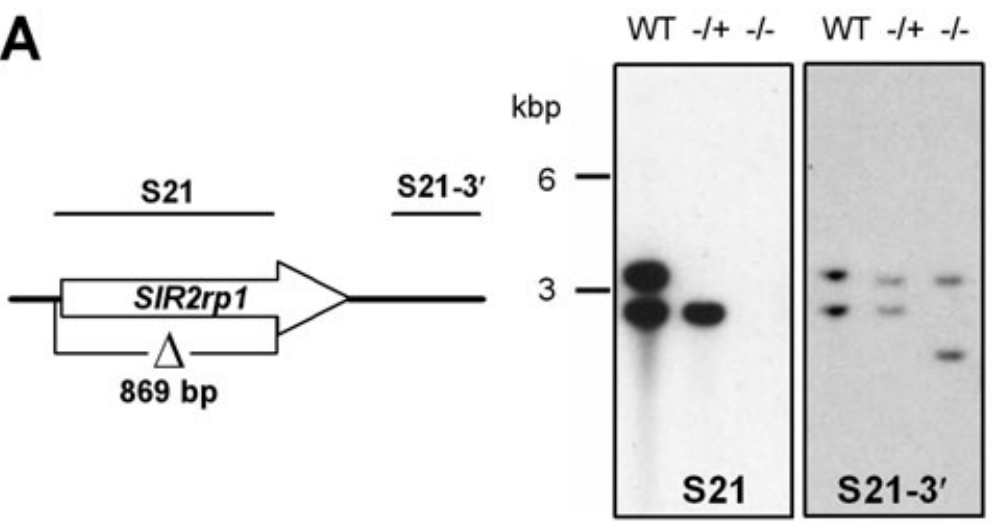

B

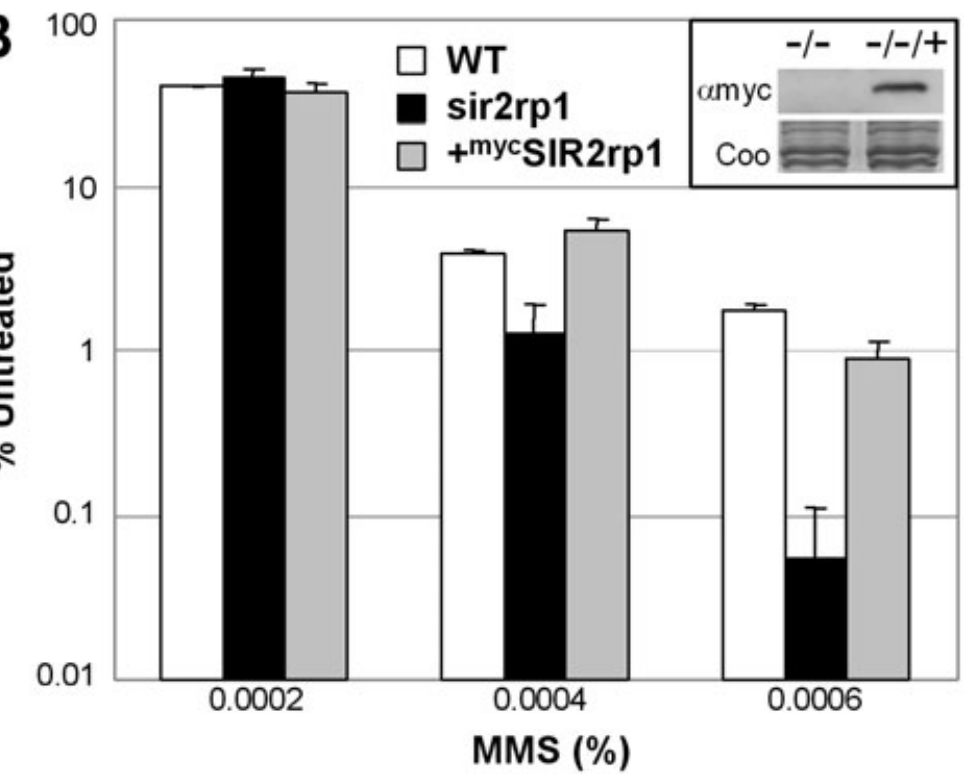

Fig. 4. SIR2rp1 controls DNA repair in bloodstream form T. brucei.

A. Schematic maps describing the gene disruption strategy (left-hand side). $\Delta$ indicates the region targeted for deletion. Probes used in Southern analysis are illustrated above the map. Gene disruption was verified by

Southern hybridization (right-hand side). Genomic DNA from wild type and transformed strains was digested with Kpnl. Both SIR2rp1 alleles are detected due to the presence of a polymorphic $\mathrm{Kpnl}$ site at this locus.

$B$. DNA repair assay. Cultures were seeded at $10^{5}$ per $\mathrm{ml}$ in triplicate in the presence of methanesulphonic acid methyl ester (MMS) at the concentration indicated and counts were taken after $32 \mathrm{~h}$. Error bars: standard error. The inset (upper panel) shows ${ }^{\text {myc }}$ SIR2rp1 expression $(-/-1+)$ in cells with both native SIR2rp1 alleles disrupted (-/-). Coo, Coomassie-stained loading control (lower panel). evidence for a direct role in telomeric silencing. We therefore turned to analysis in T. brucei.

In T. brucei, telomeric silencing interferes with RNA polymerase I (Pol I) transcription (Glover and Horn, 2006) so we monitored the expression of telomere-adjacent Pol I-driven reporter cassettes in sir2rp1 null mutants. Consistent with a role in telomeric silencing, in cells with a repressed promoter $2 \mathrm{~kb}$ from a telomere (Fig. 6A), reporter expression was increased following SIR2rp1 disruption (Fig. 6B). Northern blot analysis indicated a sixfold increase in reporter expression in sir2rp1 null mutants. In addition, sir2rp1 cells reconstituted with SIR2rp1 (Fig. 4B, inset) displayed restored repression (Fig. 6B) confirming that loss of silencing was a consequence of SIR2rp1 deficiency. Tubulin, a Pol II transcript derived from a telomere-distal gene array, appeared unaffected by SIR2rp1. Analysis of differentiated, insect-stage cells gave similar results (data not shown). Thus, our results demonstrate a role for SIR2rp1 in telomeric silencing in both major life-cycle stages. Telomeric silencing only spreads a short distance from the telomere at an RRNA locus (Glover and Horn, 2006) so we considered the idea that increased SIR2rp1 expression could extend the repressed domain. Inducible overexpression of mycSIR2rp1 failed to 'spread' silencing to a promoter $5 \mathrm{~kb}$ from an ectopic telomere at an RRNA locus (data not shown). In fact, it is Sir3, not Sir2, that is the limiting factor for the spread of silencing in S. cerevisiae (Hecht et al., 1996). Sir3 orthologues are not found beyond yeasts but our results are consistent with accessory factordependent restriction and/or spreading in T. brucei.

In T. brucei, 'basal' telomeric gene-silencing operates in both major life cycle stages. A second form of repression specifically associated with VSG gene expression sites operates only in bloodstream-form cells (Glover and Horn, 2006). The data above indicate that basal telomeric silencing is SIR2rp1-dependent. To assess the influence of SIR2rp1 on VSG-associated repression we engineered Pol I reporter cassettes upstream of a VSG221 gene (Fig. 6C) in cells with VSG221 either expressed or 


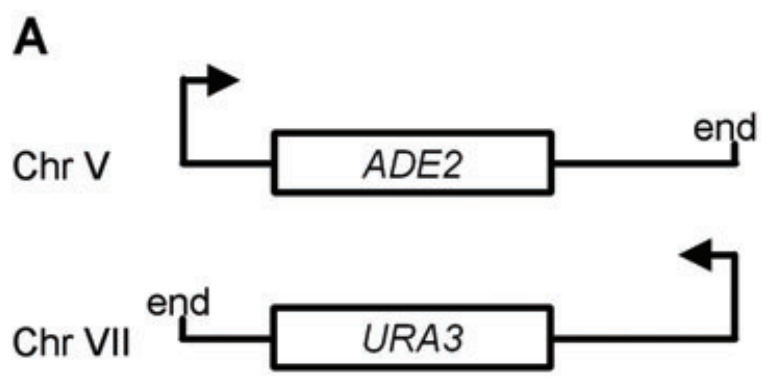

B

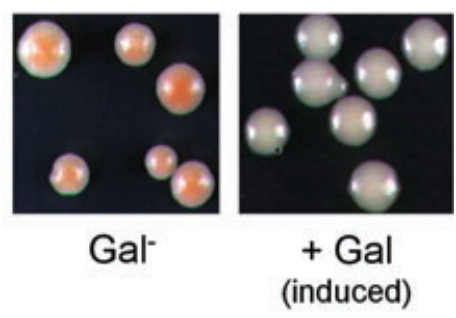

C

- Gal

+ Gal (induced)
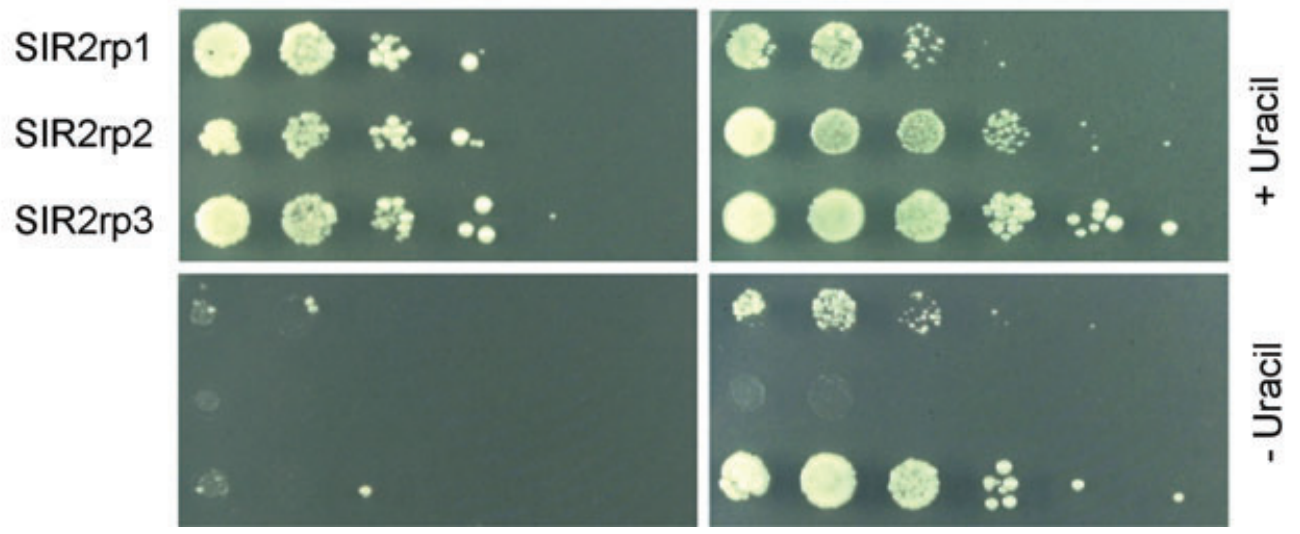

Fig. 5. SIR2rp1 disrupts telomeric silencing in S. cerevisiae.

A. The UCC3505 strain has an intact ScSIR2 gene and repressed ADE2 and URA3 reporters adjacent to telomeres on chromosomes V and VII respectively.

B. ADE2 assay. Colonies are red when $A D E 2$ is silenced and white when $A D E 2$ is expressed so the emergence of white colonies on galactose plates indicates that the telomeric $A D E 2$ gene was derepressed by SIR2rp1.

C. URA3 quantitative growth assay. Ten-fold serial dilutions. Because the telomeric URA3 gene is silenced the strain is auxotrophic for uracil (left-hand panels). SIR2rp1 expression allowed growth on plates lacking uracil confirming that SIR2rp1 disrupts telomeric silencing (right-hand panels).

repressed. The latter cells expressed VSG118 from a telomeric expression site on another chromosome and cells expressing VSG221 acted as a positive control for reporter expression. SIR2rp1 was disrupted in VSG118expressing cells and the desired disruption was confirmed by Southern blotting (data not shown). The Pol I reporter was partially (fivefold) de-repressed in sir2rp1 null mutants, as determined by northern analysis, but mRNA from the downstream VSG221 gene remained undetectable indicating strong residual repression at this locus (Fig. 6D). Thus, the SIR2rp1 effect is detected $5 \mathrm{~kb}$ from the telomere at the VSG locus but a SIR2rp1-independent mechanism maintains VSG gene silencing even when an ectopic promoter is placed just upstream. We reasoned that if VSGs were de-repressed to a sufficient level, mice would mount an immune response and eliminate the parasites after the first 'wave' of parasitaemia. We found no evidence for VSG derepression in mice, however, because the sir2rp1 null mutants established infection and, similar to wild-type cells, generated two waves of parasitaemia peaking 6 and 12 days following inoculation (data not shown).

The data above indicate maintained VSG repression in the absence of SIR2rp1. We finally assessed the ability of sir2rp1 null mutants to undergo antigenic variation and to establish VSG repression. Switching from VSG118 to VSG221 expression was previously shown to occur at a frequency of $\sim 6 \times 10^{-7}$ (Horn and Cross, 1997a) via one of two distinct mechanisms (see Fig. 7A). For these experiments, we used strains expressing VSG118 with an NPT reporter upstream of the repressed VSG221 gene and an equivalent sir2rp1 null mutant strain (see Fig. 6). To compare switching frequency in the two strains, we seeded 10 independent cultures with $\sim 10$ cells from each and, when the cultures had expanded to $1-2 \times 10^{6}$ cells, added G418 at $200 \mu \mathrm{g} \mathrm{ml}^{-1}$. The vast majority of cells were rapidly eliminated under these conditions and, importantly, at least one culture from each strain did not 
C
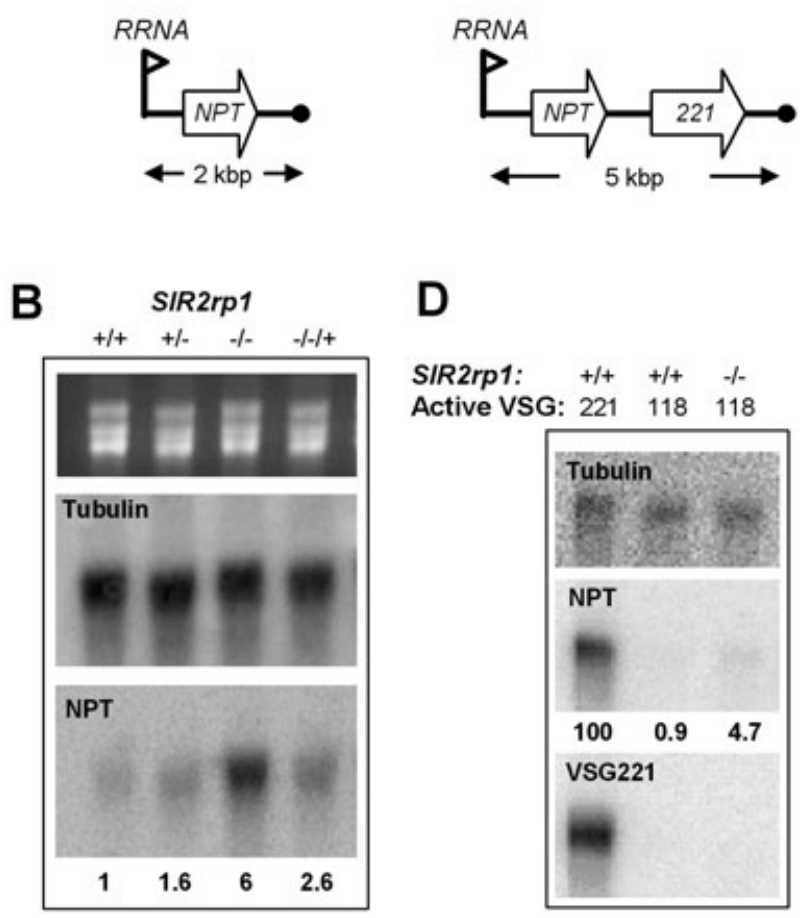

D

SIR2rp1: $\quad+/+\quad+1+\quad-/-$ Active VSG: $221 \quad 118 \quad 118$

Fig. 6. SIR2rp1 controls telomeric gene silencing.

A. An NPT gene was placed $2 \mathrm{~kb}$ from a de novo telomere (see Glover and Horn, 2006) in strains with different SIR2rp1 genotypes; +/+, wild type; +/-, heterozygous; -/-, disrupted; $-/-/+$, complemented with mycSIR2rp1 (see Fig. 4).

B. NPT ${ }^{-2}$ reporter expression was assessed by Northern blotting in bloodstream form cells. Numbers indicate normalized NPT expression as assessed by Phosphorimager analysis (Amersham). C. An NPT gene was placed $5 \mathrm{~kb}$ from a telomere within the polycistronic VSG221 expression site followed by SIR2rp1 disruption (see Fig. 4A).

D. NPT ${ }^{V S G-5}$ and VSG221 expression were assessed as in B.

recover confirming that the NPT reporter remained repressed in most cells (see Fig. 6D). After approximately 1 week, a number of G418 resistant cultures emerged. To determine whether these cultures had coactivated VSG221, we made whole-cell protein extracts and separated proteins in SDS-PAGE gels. Coomassie-staining confirmed a VSG switch in nine of the SIR2rp1 wild-type cultures (from a total of $\sim 1.8 \times 10^{7}$ cells) and in four of the sir2rp1 null mutant cultures (from a total of $\sim 1.1 \times 10^{7}$ cells). Two switches from each strain are shown in Fig. 7B. Although some of the wild-type cultures may not have been clonal, this equates to switching frequencies of $\sim 5 \times 10^{-7}$ and $\sim 3.6 \times 10^{-7}$ respectively. SIR2rp1 therefore has no significant impact on VSG switching frequency in these cells.

To determine whether sir2rp1 null mutants could establish VSG repression we needed to look for VSG221expressing cultures that retained the VSG118 gene. Southern blotting indicated that the VSG118 gene could be retained in the presence or absence of SIR2rp1 (5/6 and 2/4 switches derived from wild type and sir2rp1 null mutants respectively). Examples of VSG118 gene retention and loss, representing both mechanisms illustrated in Fig. 7A, are shown in Fig. 7C. Retention of VSG118 indicates that sir2rp1 null mutants can establish and maintain VSG118 repression concomitant with VSG221 activation.

\section{Discussion}

Sirtuins have been linked to a diverse array of biological functions. In human cells, three sirtuins localize to the nucleus, three to the mitochondria and one is microtubuleassociated (see Michishita et al., 2005). The picture is not so complex in T. brucei and our characterization of all three T. brucei SIR2rp's indicates a single nuclear protein and two mitochondrial proteins. It will be important to identify the in vivo substrates to further characterize the SIR2rp's. SIR2rp1 clusters in a phylogenetic group with the histone deacetylase, Sir2. Although the p53 deacetylase, HsSIRT1, and the tubulin deacetylase, HsSIRT2 are within the same group, our results are more consistent with histone modification by SIR2rp1 and with the restriction of SIR2rp2 and SIR2rp3 substrates to the mitochondrion.

Sir2 overexpression in S. cerevisiae is toxic (Holmes et al., 1997) and we show that SIR2rp1 overexpression in T. brucei is toxic. This may be due to inappropriate deacetylation of cytosolic substrates or hypoacetylation of nuclear substrates. Accordingly, appearance in the T. brucei cytosol may be due to saturation or loss, via deacetylation, of nuclear-binding platforms. SIR2rp1 overexpression was not shown to be toxic in insect stage T. brucei (Garcia-Salcedo et al., 2003) but this could be for either technical reasons or due to stage-regulated function. SIR2rp2 and SIR2rp3 are both mitochondrial proteins and because trypanosomatids are the most ancient branch of the eukaryal lineage to contain conventional mitochondria, our results indicate evolutionary conservation of sirtuins in this organelle. Mitochondrial import may rely upon $A r g$ residues $\left(R^{4,16}\right.$ in TbSIR2RP2, and $\mathrm{R}^{2,3,6}$ in $\mathrm{TbSIR} 2 \mathrm{RP} 3$ ) close to the $\mathrm{N}$-terminus (see Hausler et al., 1997). It is now clear that sirtuins can modulate the $\mathrm{NAD}^{+}: \mathrm{NADH}$ ratio, nicotinamide and O-acetyl-ADPribose levels and these small molecules can in turn influence sirtuin activity. In some cases, the sirtuins may be considered as metabolic sensors, linking cellular energy status to deacetylation and/or ADP ribosylation activity. The presence of two sirtuins in the T. brucei mitochondrion is consistent with conserved roles in energy homeostasis, as is conserved sirtuin-dependent acetylation of acetyl-CoA synthetase (AceCS) (Hallows et al., 2006; Schwer et al., 2006) and ADP-ribosylation of glutamate dehydrogenase (GDH) (Haigis et al., 2006). AceCS and 
A

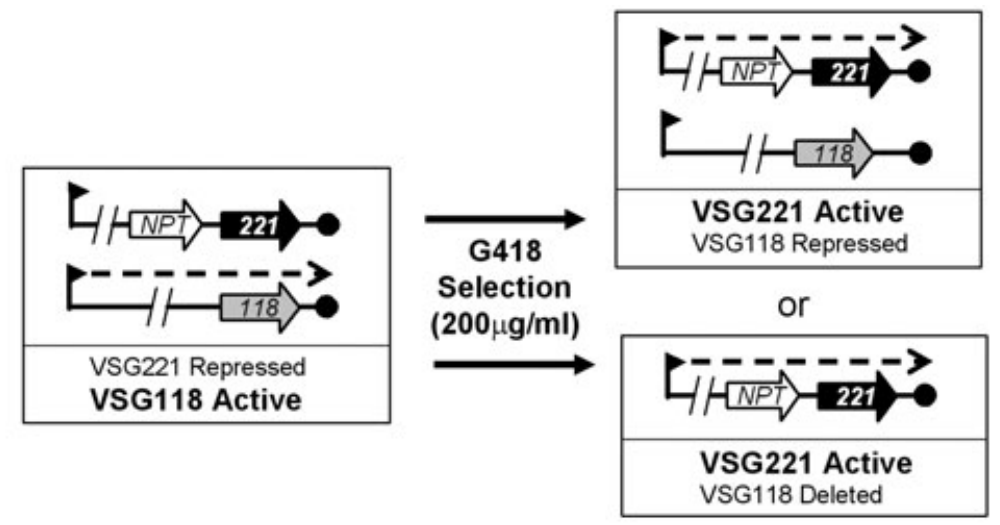

Fig. 7. SIR2rp1 is not required for antigenic variation.

A. The schematic illustrates the expected VSG-switching mechanisms underlying increased G418 resistance/VSG switching. B. Protein extracts from parental (118) cells and two 'switched' clones for each cell type were separated by SDS-PAGE and stained with Coomassie. VSG118 migrates at $\sim 60 \mathrm{kDa}$ while VSG221 migrates at $\sim 55 \mathrm{kDa}$. Arrows indicate the abundant VSGs and the selectable reporter (NPT) which is also abundant when activated.

C. Southern blotting with a VSG118 probe indicates that both SIR2rp1 wild-type cells and sir2rp1 null mutants could inactivate VSG118 with retention or loss of VSG118 as illustrated in A.

B

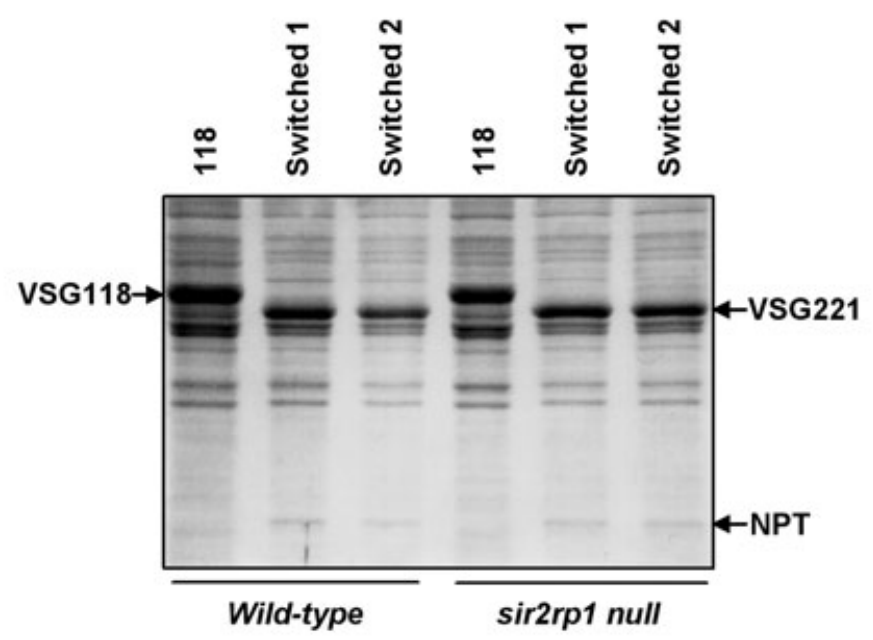

C

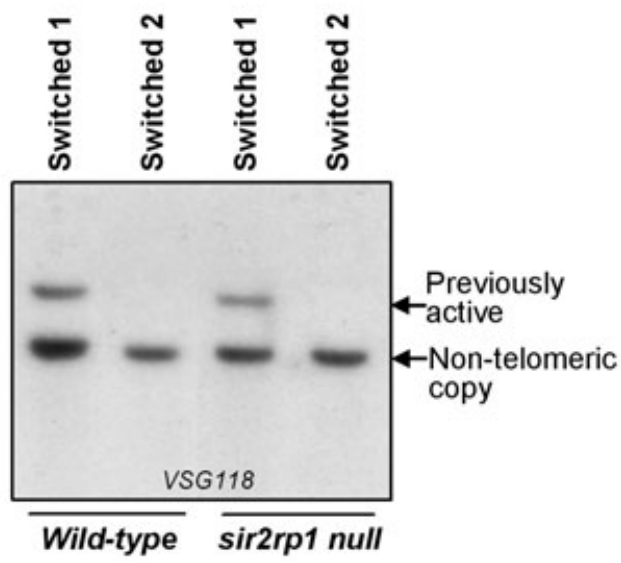

GDH are mitochondrial enzymes activated by deacetylation and inhibited by ADP-ribosylation, respectively, and similar pathways mediated by SIR2rp2 and/or SIR2rp3 may operate in trypanosomatids.

Primary ADP-ribosylation activity and a role in DNA repair was reported for SIR2rp1 in insect stage T. brucei cells in which SIR2rp1 was expressed at approximately double or one third the wild-type level (Garcia-Salcedo et al., 2003). Using MMS, an alkylating agent that generates DNA lesions, we confirmed a role for SIR2rp1 in DNA repair in insect stage cells and demonstrated a similar role in mammal-infective bloodstream form cells. A role for sirtuins in DNA repair may be conserved from trypanosomatids to mammals since mice lacking Sirt6, another sirtuin with primary ADP-ribosylation activity (Liszt et al., 2005), also display MMS hypersensitivity (Mostoslavsky 
et al., 2006). As homologous recombination is important for antigenic variation it will be interesting to determine whether SIR2rp1 can impact on VSG-recombinationrelated repair mechanisms.

Saccharomyces cerevisiae Sir2 is required for telomeric gene silencing (Rusche et al., 2003) and even an archael Sir2 orthologue appears to repress transcription by deacetylating the chromatin protein, Alba (Bell et al., 2002). Sir2 orthologues have also been shown to repress expression of native subtelomeric genes as demonstrated by phenotypic switching in Candida albicans (PerezMartin et al., 1999) and antigenic variation in P. falciparum (Figueiredo and Scherf, 2005). In the mammalian host bloodstream, T. brucei undergoes antigenic variation. This requires mutually exclusive Pol l-dependent expression of a single VSG gene and the silencing of hundreds of subtelomeric VSG genes, about 20 of which are located downstream of intact promoters. We previously reported 'basal' telomeric Pol I silencing in both major life-cycle stages of $T$. brucei whereas the form of repression that may control antigenic variation is bloodstream form specific (Glover and Horn, 2006). Here, we have shown that SIR2rp1, the only nuclear SIR2rp in T. brucei, controls basal telomeric silencing but appears to have little impact on VSG expression site associated repression. sir2rp1 null mutants maintain repression of a telomere-adjacent $V S G$, they escape an immune response in mice and they undergo antigenic variation. Thus, the current results indicate two mechanistically distinct forms of repression: SIR2rp1-dependent telomeric silencing and SIR2rp1independent VSG silencing. We cannot rule out a role for SIR2rp1 in tight VSG repression in natural infections due to the telomere-proximal location of many VSGs and the contribution of SIR2rp1 to telomeric silencing demonstrated here. In addition, a set of VSG genes are transcribed from promoters $\sim 5 \mathrm{~kb}$ from the telomere during the first week of a mammalian infection (see Horn and Barry, 2005). It is possible that these 'metacyclic' VSG expression sites are repressed in a SIR2rp1-dependent manner in the insect-stage. Other factors, such as class I/II type deacetylases (Ingram and Horn, 2002) or other histone modifying enzymes and remodelling factors (see table S4 in Ivens et al., 2005) may have a more significant impact on VSG gene expression and silencing in bloodstream-form cells.

As is the case for TbSIR2rp1, most sirtuins are dispensable. For example, yeast strains lacking Sir2 and all four Sir2 paralogues are viable (Smith et al., 2000). We were able to generate $T$. brucei sir2rp1 null mutants which displayed neither growth nor differentiation defects. Leishmania SIR2rp1 however, has been reported to be essential for growth (Vergnes et al., 2005) and was also reported to be found in cytoplasmic granules and to be excreted (Zemzoumi et al., 1998). Although the T. brucei and Leishmania proteins are conserved throughout and appear to be homologues (59\% identity and $75 \%$ similarity), these features contrast with the dispensability and conserved role in DNA repair and transcriptional silencing of TbSIR2rp1 reported here. Further studies may help to determine whether the two proteins really have such apparently distinct functions.

Histone deacetylases and chromatin modifiers have potentially major roles in controlling gene expression in important human and animal pathogens such as the trypanosomatids. Although some pathogens have co-opted sirtuins to repress subtelomeric virulence factor genes, $T$. brucei has adopted a different, or at least modified, strategy to control the VSG repertoire. However, sirtuin-dependent telomeric silencing in $T$. brucei does suggest widespread conservation of this particular phenomenon. Similar to Sir2 in budding yeast, TbSIR2rp1 not only represses transcription immediately adjacent to telomeres but also, presumably through relocalization to sites of DNA damage (Martin et al., 1999), controls DNA repair more broadly across the genome. Although SIR2rp1 substrates have not been defined, it is tempting to speculate that this sirtuin represses transcription at telomeres and promotes repair via chromatin modification.

\section{Experimental procedures}

Trypanosoma brucei growth and manipulation

All cells were derived from Lister 427 bloodstream forms MITat1.2 (clone 221a) and MITat1.5 (clone 118a), grown in $\mathrm{HMI}-11$, transformed with linear DNA constructs and differentiated to the insect stage as previously described (see Alsford et al., 2005). For expression of tagged proteins, recombinant vectors were integrated at RRNA loci in T. brucei cells expressing T7 RNA polymerase (T7RNAp) and/or tetracycline-repressor (TetR) (Wirtz et al., 1999; Alsford et al., 2005). Cells expressing T7RNAp were not used for reporter assays (Figs 6 and 7) because this polymerase can compromise reporter cassette silencing (data not shown). Drugs were added $\sim 6 \mathrm{~h}$ post transfection at the following concentrations: blasticidin (Invitrogen), $10 \mu \mathrm{g} \mathrm{ml}^{-1}$; puromycin (Calbiochem), $2 \mu \mathrm{g} \mathrm{ml}^{-1}$; G418 (MBI Fermentas), $2 \mu \mathrm{g} \mathrm{ml}^{-1}$; phleomycin (CayLa), $2 \mu \mathrm{g} \mathrm{m}^{-1}$; hygromycin (Sigma), $2.5 \mu \mathrm{g} \mathrm{ml}^{-1}$. Cell counts were carried out using a haemocytometer. Tetracycline and MMS were from Sigma.

\section{SIR2RP cloning}

All three SIR2rp genes used in this study were derived from the Lister 427 T. brucei strain. We isolated the SIR2rp1 gene first using degenerate oligonucleotides; SIR/PF (5'-GGIRTICCIGAYTTYMG-3') and SIR20 (5'-TCIARIIYR TCDATRTTYTG-3') (D: A/T/G, I: inosine, M: A/C, R: A/G, Y: $\mathrm{C} / \mathrm{T})$; that recognize sequence encoding conserved motifs in SIR2rp's (GI/VPDFR and QNIDG/T/NLE respectively; see 
Fig. 1). Temperature was cycled 30 times through 94, 50 and $72^{\circ} \mathrm{C}$ for $30 \mathrm{~s}$ each, in the presence of $100 \mathrm{ng}$ T. brucei genomic DNA and Taq DNA polymerase (MBI Fermentas). A $\sim 250$ bp product was purified and cloned into a pGem-T vector (Promega). Clones that hybridized with the PCR product, representing both alleles, were isolated from a T. brucei genomic library consisting of $\mathrm{Kpnl}$ fragments in pBluescript (Stratagene). SIR2rp2 and SIR2rp3 (along with SIR2rp1) were subsequently identified in the T. brucei genome sequence (see table S4 in Ivens et al., 2005).

\section{Plasmid constructs}

Genes or gene fragments were amplified by PCR from genomic DNA using Vent high fidelity DNA polymerase (New England Biolabs) in conjunction with specific primer pairs (relevant restriction enzyme sites are underlined and start and stop codons are indicated in lower-case) unless stated otherwise. For epitope-tagging constructs, SIR2rp tagging primers were as follows:

i) N-terminal tagging

- SIR2rp1: GCTCTAGAACAGAACCGAAGTTAGCAAC and CGGGATCCttaACCCTCAACGACTTTTTC

- SIR2rp2: GCTCTAGAGCTGACCGCCTTGCTATTT and CGGGATCCttaCTTCGTGTCGCCCAAC

- SIR2rp3: GCTCTAGAAGGCGGCCCAATCGTATG and CGGGATCCtcaTGCCGCGGGACCCTT

ii) C-terminal tagging

- SIR2rp2: GATCAAGCTatgGCTGACCGCCTTGCTAT and GATCTCTAGACTTCGTGTCGCCCAACTCGA

- SIR2rp3: GATCAAGCTTatgAGGCGGCCCAATCGTAT and GATCTCTAGATGCCGCGGGACCCTTTCCGT

The open reading frames (ORFs) were then cloned into the tetracycline-inducible $\mathrm{N}$ - or C-terminal tagging (cMyc or eGFP) vectors of the pT7, pT7a and pRPa series (Alsford et al., 2005). To generate the SIR2rp1 gene disruption constructs, we used the SIR2rp1 genomic clone (-915 nt to $+631 \mathrm{nt}$ relative to the ORF). An $873 \mathrm{bp}$ Sall/Spel fragment was replaced using NPT, BLE, HYG or BLA selectable markers. For expression in yeast, we used the following primers: SIR2rp1, GGAATTCCATatgACAGAACCGAAGTT AGC and CGGGATCCttaACCCTCAACGACTTTTTC SIR2rp2, CCTCGAGTGCAGTTGAGTGATatgG and CGAG CTCGGATCCTTCATGTCACTTGGCAC SIR2rp3, CCTC GAGGACTGTTTGTAGGTTCAAC and CGGATCCAGC CACTCAAACTGCAAC. The ORFs were cloned in a galactose-inducible expression vector, pTCG, a high-copy vector ( $2 \mu$ origin of replication) with a Trp selectable marker. pRn5 was used to integrate a reporter cassette at the VSG221 locus. This construct was derived from pbRn5 (Horn and Cross, 1997b) by removing the BLE gene.

\section{RNA and DNA analysis}

DNA sequencing was performed using a Thermo Sequenase dye terminator kit (Applied Biosystems), a thermal cycler and an $\mathrm{ABI}$ Prism 377 automated sequencer according to the manufacturer instructions. PCR, RT-PCR, Southern and northern analysis were all carried out according to standard protocols. Signals on Northern blots were quantified using a Phosphorimager (Amersham).

\section{Protein analysis}

For Western blotting whole cell lysates were separated by SDS-PAGE and electroblotted using standard protocols. Western blots were developed using mouse monoclonal anticMyc (4A6, Upstate or 9E10, Santa Cruz) or rabbit polyclonal anti-GFP (Molecular Probes) according to the manufacturers' instructions. Signals were detected using an $E C L+k i t$ (Amersham).

For fluorescence microscopy, cells were fixed in $2 \%(\mathrm{v} / \mathrm{v})$ paraformaldehyde in phosphate buffered saline (PBS) at $4^{\circ} \mathrm{C}$ for at least $1 \mathrm{~h}$, dried onto glass slides following washing in ice cold PBS and $1 \%(\mathrm{w} / \mathrm{v}) \mathrm{BSA}$ in water, permeabilized in $0.5 \%(\mathrm{v} / \mathrm{v})$ Triton-X100 in PBS for $20 \mathrm{~min}$ and blocked in 50\% (v/v) foetal bovine serum in PBS for $10 \mathrm{~min}$. Indirect detection of cMyc and eGFP-tagged proteins was carried out using mouse monoclonal anti-cMyc (9E10, Santa Cruz) and polyclonal rabbit anti-GFP (Molecular Probes). Antibody incubations were carried out according to manufacturers' instructions. Cells were mounted in Vectashield (Vector Laboratories) containing the DNA counterstain, 4',6-diamidino-2phenylindole (DAPI). Slides were analysed on a Nikon Eclipse E600 epifluorescence microscope. Differential interference contrast (DIC) and fluorescence images were captured using a Coolsnap FX (Photometrics) CCD camera and processed in Metamorph 5.0 (Universal Imaging) and Photoshop Elements 2.0 (Adobe). To visualize the mitochondrion, cells were incubated with $100 \mathrm{nM}$ MitoTracker Red CMXRos (Molecular Probes) in $\mathrm{HMl}-11$ at $37^{\circ} \mathrm{C}$ for $5 \mathrm{~min}$ prior to fixation.

\section{Yeast assays}

Saccharomyces cerevisiae strains UCC2 and UCC3505 were grown at $30^{\circ} \mathrm{C}$ and transformed using the lithium acetate procedure according to standard protocols (see Singer and Gottschling, 1994). For SIR2rp induction, glucose was replaced with $3 \%$ galactose and cells were pregrown for 4 days at $30^{\circ} \mathrm{C}$. For the $A D E 2$ assay, adenine hemisulphate was at $20 \mu \mathrm{g} \mathrm{ml}^{-1}$ and colonies were incubated for 3 weeks at $4^{\circ} \mathrm{C}$ for full colour development. For the URA3 assay, 10 -fold serial dilutions in $\mathrm{H}_{2} \mathrm{O}$ were spotted on plates and grown for 4 days.

\section{Acknowledgements}

This work was supported by a Research Career Development Fellowship to DH (052323) and a Project Grant (069909) both from The Wellcome Trust. We thank Alexandra Ingram (LSHTM) for assistance, Daniel Gottschling (Fred Hutchinson Cancer Research Centre, Seattle, USA) for providing the yeast expression plasmid, pTCG, and the yeast strains, UCC2 and UCC3505, and Keith Gull (University of Oxford, Oxford, UK) for the Ac-tubulin antibody, C3B9. We are also grateful to Martin Taylor (LSHTM) for helpful comments on the manuscript. 


\section{References}

Alsford, S., Kawahara, T., Glover, L., and Horn, D. (2005) Tagging a T. brucei RRNA locus improves stable transfection efficiency and circumvents inducible expression position effects. Mol Biochem Parasitol 144: 142-148.

Bell, S.D., Botting, C.H., Wardleworth, B.N., Jackson, S.P., and White, M.F. (2002) The interaction of Alba, a conserved archaeal chromatin protein, with Sir2 and its regulation by acetylation. Science 296: 148-151.

Bitterman, K.J., Anderson, R.M., Cohen, H.Y., LatorreEsteves, M., and Sinclair, D.A. (2002) Inhibition of silencing and accelerated aging by nicotinamide, a putative negative regulator of yeast sir2 and human SIRT1. J Biol Chem 277: 45099-45107.

Blander, G., and Guarente, L. (2004) The sir2 family of protein deacetylases. Annu Rev Biochem 73: 417-435.

Chang, J.H., Kim, H.C., Hwang, K.Y., Lee, J.W., Jackson, S.P., Bell, S.D., and Cho, Y. (2002) Structural basis for the NAD-dependent deacetylase mechanism of Sir2. J Biol Chem 277: 34489-34498.

Chen, X.J., and Clark Walker, G.D. (1994) sir2 mutants of Kluyveromyces lactis are hypersensitive to DNA-targeting drugs. Mol Cell Biol 14: 4501-4508.

Cockell, M.M., Perrod, S., and Gasser, S.M. (2000) Analysis of Sir2p domains required for rDNA and telomeric silencing in Saccharomyces cerevisiae. Genetics 154: 1069-1083.

Figueiredo, L., and Scherf, A. (2005) Plasmodium telomeres and telomerase: the usual actors in an unusual scenario. Chromosome Res 13: 517-524.

Frye, R.A. (2000) Phylogenetic classification of prokaryotic and eukaryotic Sir2-like proteins. Biochem Biophys Res Comm 273: 793-798.

Garcia-Salcedo, J.A., Gijon, P., Nolan, D.P., Tebabi, P., and Pays, E. (2003) A chromosomal SIR2 homologue with both histone NAD-dependent ADP-ribosyltransferase and deacetylase activities is involved in DNA repair in Trypanosoma brucei. EMBO J 22: 5851-5862.

Glover, L., and Horn, D. (2006) Repression of polymerase I-mediated gene expression at Trypanosoma brucei telomeres. EMBO Rep 7: 93-99.

Gottlieb, S., and Esposito, R.E. (1989) A new role for a yeast transcriptional silencer gene, SIR2, in regulation of recombination in ribosomal DNA. Cell 56: 771-776.

Haigis, M.C., Mostoslavsky, R., Haigis, K.M., Fahie, K., Christodoulou, D.C., Murphy, A.J., et al. (2006) SIRT4 inhibits glutamate dehydrogenase and opposes the effects of calorie restriction in pancreatic $\beta$ cells. Cell 126: 941954.

Hallows, W.C., Lee, S., and Denu, J.M. (2006) Sirtuins deacetylate and activate mammalian acetyl-CoA synthetases. Proc Natl Acad Sci USA 103: 10230-10235.

Hausler, T., Stierhof, Y.D., Blattner, J., and Clayton, C. (1997) Conservation of mitochondrial targeting sequence function in mitochondrial and hydrogenosomal proteins from the early-branching eukaryotes Crithidia, Trypanosoma and Trichomonas. Eur J Cell Biol 73: 240251.

Hecht, A., Strahl-Bolsinger, S., and Grunstein, M. (1996) Spreading of transcriptional repressor SIR3 from telomeric heterochromatin. Nature 383: 92-96.
Holmes, S.G., Rose, A.B., Steuerle, K., Saez, E., Sayegh, S., Lee, Y.M., and Broach, J.R. (1997) Hyperactivation of the silencing proteins, Sir2p and Sir3p, causes chromosome loss. Genetics 145: 605-614.

Horn, D., and Cross, G.A. (1997a) Analysis of Trypanosoma brucei vsg expression site switching in vitro. Mol Biochem Parasitol 84: 189-201.

Horn, D., and Cross, G.A. (1997b) Position-dependent and promoter-specific regulation of gene expression in Trypanosoma brucei. EMBO J 16: 7422-7431.

Horn, D., and Barry, J.D. (2005) The central roles of telomeres and subtelomeres in antigenic variation in African trypanosomes. Chromosome Res 13: 525-533.

Imai, S., Armstrong, C.M., Kaeberlein, M., and Guarente, L. (2000) Transcriptional silencing and longevity protein Sir2 is an NAD-dependent histone deacetylase. Nature 403: 795-800.

Ingram, A.K., and Horn, D. (2002) Histone deacetylases in Trypanosoma brucei: two are essential and another is required for normal cell cycle progression. Mol Microbiol 45: 89-97.

Ivens, A.C., Peacock, C.S., Worthey, E.A., Murphy, L., Aggarwal, G., Berriman, M., et al. (2005) The genome of the kinetoplastid parasite, Leishmania major. Science 309: 436-442.

Janzen, C.J., Fernandez, J.P., Deng, H., Diaz, R., Hake, S.B., and Cross, G.A. (2006) Unusual histone modifications in Trypanosoma brucei. FEBS Lett 580: 2306-2310.

Liszt, G., Ford, E., Kurtev, M., and Guarente, L. (2005) Mouse Sir2 homolog SIRT6 is a nuclear ADPribosyltransferase. J Biol Chem 280: 21313-21320.

Longtine, M.S., Enomoto, S., Finstad, S.L., and Berman, J. (1993) Telomere-mediated plasmid segregation in Saccharomyces cerevisiae involves gene products required for transcriptional repression at silencers and telomeres. Genetics 133: 171-182.

Luo, J., Nikolaev, A.Y., Imai, S., Chen, D., Su, F., Shiloh, A., et al. (2001) Negative control of p53 by Sir2 promotes cell survival under stress. Cell 107: 137-148.

Martin, S.G., Laroche, T., Suka, N., Grunstein, M., and Gasser, S.M. (1999) Relocalization of telomeric Ku and SIR proteins in response to DNA strand breaks in yeast. Cell 97: 621-633.

Michishita, E., Park, J.Y., Burneskis, J.M., Barrett, J.C., and Horikawa, I. (2005) Evolutionarily conserved and nonconserved cellular localizations and functions of human SIRT proteins. Mol Biol Cell 16: 4623-4635.

Min, J., Landry, J., Sternglanz, R., and Xu, R.M. (2001) Crystal structure of a SIR2 homolog-NAD complex. Cell 105: 269-279.

Mostoslavsky, R., Chua, K.F., Lombard, D.B., Pang, W.W., Fischer, M.R., Gellon, L., et al. (2006) Genomic instability and aging-like phenotype in the absence of mammalian SIRT6. Cell 124: 315-329.

Muth, V., Nadaud, S., Grummt, I., and Voit, R. (2001) Acetylation of TAF,68, a subunit of TIF-IB/SL1, activates RNA polymerase I transcription. EMBO J 20: 1353-1362.

North, B.J., Marshall, B.L., Borra, M.T., Denu, J.M., and Verdin, E. (2003) The human Sir2 ortholog, SIRT2, is an $\mathrm{NAD}^{+}$-dependent tubulin deacetylase. Mol Cell 11: 437444. 
Pappas, D.L., Jr, Frisch, R., and Weinreich, M. (2004) The $N A D^{+}$-dependent Sir2p histone deacetylase is a negative regulator of chromosomal DNA replication. Genes Dev 18: 769-781.

Perez-Martin, J., Uria, J.A., and Johnson, A.D. (1999) Phenotypic switching in Candida albicans is controlled by a SIR2 gene. EMBO J 18: 2580-2592.

Perrod, S., Cockell, M.M., Laroche, T., Renauld, H., Ducrest, A., Bonnard, C., and Gasser, M. (2001) A cytosolic NADdependent deacetylase, Hst2p, can modulate nucleolar and telomeric silencing in yeast. EMBO J 20: 197-209.

Proudfoot, C., and McCulloch, R. (2005) Distinct roles for two RAD51-related genes in Trypanosoma brucei antigenic variation. Nucleic Acids Res 33: 6906-6919.

Rusche, L.N., Kirchmaier, A.L., and Rine, J. (2003) The establishment, inheritance, and function of silenced chromatin in Saccharomyces cerevisiae. Annu Rev Biochem 72: 481-516.

Sasse, R., and Gull, K. (1988) Tubulin post-translational modifications and the construction of microtubular organelles in Trypanosoma brucei. J Cell Sci 90: 577589.

Schwer, B., North, B.J., Frye, R.A., Ott, M., and Verdin, E. (2002) The human silent information regulator (Sir)2 homologue hSIRT3 is a mitochondrial nicotinamide adenine dinucleotide-dependent deacetylase. J Cell Biol 158: 647657.

Schwer, B., Bunkenborg, J., Verdin, R.O., Andersen, J.S., and Verdin, E. (2006) Reversible lysine acetylation controls the activity of the mitochondrial enzyme acetyl-CoA synthetase 2. Proc Natl Acad Sci USA 103: 1022410229.

Singer, M.S., and Gottschling, D.E. (1994) TLC1: template RNA component of Saccharomyces cerevisiae telomerase. Science 266: 404-409.

Smith, J.S., Brachmann, C.B., Celic, I., Kenna, M.A., Muhammad, S., Starai, V.J., et al. (2000) A phylogenetically conserved $\mathrm{NAD}^{+}$-dependent protein deacetylase activity in the
Sir2 protein family. Proc Natl Acad Sci USA 97: 66586663.

Starai, V.J., Celic, I., Cole, R.N., Boeke, J.D., and EscalanteSemerena, J.C. (2002) Sir2-dependent activation of acetylCoA synthetase by deacetylation of active lysine. Science 298: 2390-2392.

Tanner, K.G., Landry, J., Sternglanz, R., and Denu, J.M. (2000) Silent information regulator 2 family of NADdependent histone/protein deacetylases generates a unique product, 1-O-acetyl-ADP-ribose. Proc Natl Acad Sci USA 97: 14178-14182.

Tsang, A.W., and Escalante-Semerena, J.C. (1998) CobB, a new member of the SIR2 family of eucaryotic regulatory proteins, is required to compensate for the lack of nicotinate mononucleotide: 5,6-dimethylbenzimidazole phosphoribosyltransferase activity in cobT mutants during cobalamin biosynthesis in Salmonella typhimurium LT2. J Biol Chem 273: 31788-31794.

Tsukamoto, Y., Kato, J., and Ikeda, H. (1997) Silencing factors participate in DNA repair and recombination in Saccharomyces cerevisiae. Nature 388: 900-903.

Vaziri, H., Dessain, S.K., Ng Eaton, E., Imai, S.I., Frye, R.A., Pandita, T.K., et al. (2001) hSIR2 (SIRT1) functions as an NAD-dependent p53 deacetylase. Cell 107: 149159.

Vergnes, B., Sereno, D., Tavares, J., Cordeiro-da-Silva, A., Vanhille, L., et al. (2005) Targeted disruption of cytosolic SIR2 deacetylase discloses its essential role in Leishmania survival and proliferation. Gene 363: 85-96.

Wirtz, E., Leal, S., Ochatt, C., and Cross, G.A. (1999) A tightly regulated inducible expression system for conditional gene knock-outs and dominant-negative genetics in Trypanosoma brucei. Mol Biochem Parasitol 99: 89-101.

Zemzoumi, K., Sereno, D., Francois, C., Guilvard, E., Lemesre, J.L., and Ouaissi, A. (1998) Leishmania major. cell type dependent distribution of a $43 \mathrm{kDa}$ antigen related to silent information regulatory-2 protein family. Biol Cell 90: 239-245. 